\title{
Optimización del Proceso de Innovación para Proyectos Internos en las Empresas
}

\author{
Davi N. da Silva ${ }^{(1)}$, Raimundo K. Vieira(1), Adalena K. Vieira(1) y Miriam de Santiago ${ }^{(2)}$ \\ (1) Universidad Federal de Amazonas (UFAM), Departamento de Posgrado en Ingeniaría de Producción, \\ calle General Rodrigo Octávio Jordán Ramos, No.6200, Manaus, Brasil (e-mail: davidns15@hotmail.com; \\ kennedy71@gmail.com; adalenakennedy@gmail.com) \\ (2) Palm Beach State College, Departamento de Diseño, calle Congress Ave No. 4200S, Lake Worth, \\ Flórida, USA (e-mail: designermiriamds@gmail.com)
}

Recibido Sep. 22, 2015; Aceptado Nov. 11, 2015; Versión final Ene. 10, 2016, Publicado Jun. 2016

\begin{abstract}
Resumen
Este trabajo aborda el problema de innovación de proyectos internos con el objetivo de desarrollar el proceso de innovación de productos industriales. Para estos efectos se presenta una metodología de mejora en el modelo de innovación de una empresa privada de innovación y servicios tecnológicos, llamada Servicio Nacional de Aprendizaje Industrial (SENAI), ubicada en Manaus, Amazonas (Brasil). Para lograr esto se llevó a cabo una revisión de la literatura sobre el tema de innovación y para modificar la estrategia de innovación se utilizó de un estudio con los quince proyectos presentados por el SENAI en el año de 2014 a través del modelo de innovación abierta. El análisis del modelo de innovación abierta y el estudio de casos permitieron detectar los principales problemas, lo que posibilitó la creación de un nuevo modelo con quince etapas para trabajar la innovación. Se concluye que el modelo propuesto ha permitido mejorar la gestión de proyectos de innovación y que el modelo puede ser utilizado por todas las empresas.
\end{abstract}

Palabras clave: modelos de innovación; proyectos; administración; tecnología; estrategias de innovación

\section{Optimization of the Innovation Process for Internal Projects in Companies}

\begin{abstract}
This paper addresses the problem of internal innovation projects with the aim of developing the innovation process of industrial products. To this end a methodology for improving the innovation model of a private company for innovation and technology services, named National Service of Industrial Learning (SENAI), located in Manaus, Amazonas (Brazil) is presented. To achieve this we conducted a literature review on the topic of innovation and to modify the innovation strategy a study with fifteen projects presented by SENAI in the year 2014 using the open innovation model was used. The analysis of the open innovation model and case studies allowed detecting the main problems, which allowed the creation of a new model with fifteen stages of innovation to work on innovation. It is concluded that the proposed model has allowed improving the management of innovation projects and that the model can be used by any company.
\end{abstract}

Keywords: innovation model; projects; management; technology; innovation strategies 


\section{INTRODUCCIÓN}

La palabra innovación se ha utilizado en todo el mundo y en todos los sectores de la sociedad moderna. Está conectada a la competitividad y la supervivencia de las organizaciones, un hecho que muestra actualmente la necesidad de formar personas cada vez más creativas y conocedoras de las herramientas innovadoras. Para Von Hippel (2012), en una economía donde hay una ausencia de un sector industrial desarrollado, los profesionales deben estar preparados con capacidades diferenciadas e innovadoras que realmente marcan la diferencia. En el caso específico de la aplicabilidad es importante saber que la innovación también cubre el ambiente profesional, afectando directamente a los funcionarios que trabajan con proyectos. Para Mihaela y Titán (2014), tiene que haber una política coherente de innovación, donde el proceso de desarrollo de una nación consiste en un trípode - la educación, la investigación y la innovación.

La innovación en general debe ser la conexión entre la teoría y la práctica. Es necesario definir lo que las organizaciones realmente quieren y lo que las organizaciones consideran importante. Para Mahdi et al. (2015), las actividades prácticas producen la motivación ideal para que los funcionarios de las empresas se vuelvan fuertes, motivados y seguros. Esta es la nueva tendencia de la innovación, trabajar proyectos con el apoyo de organizaciones y empresas.

Este trabajo se centra en la optimización del proceso de desarrollo e innovación de productos industriales e ambientales de una empresa privada de innovación, servicios tecnológicos y de enseñanza profesional, el SENAI, localizada en el Brasil, en la ciudad de Manaus. EI SENAI tiene la siguiente misión: Promover la educación profesional y tecnológica, la innovación y la transferencia de tecnologías industriales, contribuyendo para aumentar la competitividad de la industria brasileña. El SENAI cumple con las necesidades demandadas por las empresas del segmento de electrónica, motocicleta, material plástico y metal-mecánica del Polo Industrial de Manaus (PIM), promoviendo así la innovación. En el ano de 2014 fueron desarrollados 15 proyectos internos de innovación por los equipos del SENAI y ninguno de estos proyectos llegó a ser utilizado por las industrias. Cabe destacar que estos problemas afectan directamente la competitividad de las empresas, lo que en lo largo incide en una disminución de las competencias técnicas, razón por la cual es de interés buscar alternativas de solución al problema expuesto.

La idea de proyectos internos con una metodología activa con acceso teórico y práctico a las actividades de carácter innovador y creativo que permiten a los funcionarios de las empresas obtener esta competencia técnica se pide cada vez más. Para Mâţă y Suciu (2014), un número creciente de países están siguiendo los modelos de las reformas de otros países que ya han alcanzado el éxito con esta alineación entre la teoría y la práctica. Debe haber una conexión entre las empresas y las instituciones que trabajan con innovación. Para Genis-Gruber y Öğüt (2014), la variedad de demandas de los clientes obliga a empresa a ser más innovadora, abriendo oportunidades de alianzas estratégicas para la promoción de la teoría y práctica.

Este enfoque debe ser entendido como una nueva metodología, la integración de un nuevo punto de vista que se establecerá en todo el mundo. Para Gündoğdu (2012), debemos repensar los proyectos internos de innovación debido a que el avance de la información y la comunicación en las últimas décadas han llevado a las empresas a quedarse mucho más cerca de los clientes y responder más rápidamente a sus necesidades. Para Seman et al. (2012), los proyectos que se utilizan ampliamente en el desarrollo de un nuevo producto pueden estimular indirectamente la creación de más innovación, o sea, la innovación de otros productos, la innovación de procesos de producción y manufactura, gestión de la innovación y la innovación de mercadeo.

Por lo tanto, el propósito de este estudio es explorar los factores que influyen en la generación de proyectos internos de innovación de las empresas y de esto se presenta una nueva herramienta innovadora que puede ser utilizada por todas las empresas que deseen desarrollar su proceso de innovación interna y externa. Para lograr esto, también se llevó a cabo una revisión de la literatura sobre el tema de innovación. Así, este estudio examina la relación entre la cultura organizacional de una empresa privada de innovación, servicios tecnológicos y de enseñanza profesional y la orientación de la innovación que ocurre en las empresas. Finalmente, concluimos con nuestros pensamientos sobre las consecuencias administrativas del nuevo modelo de innovación al alcance de todos.

\section{DESCRIPCIÓN DEL MÉTODO}

El estudio de caso se llevó a cabo en el SENAI Waldemiro Lustoza - Unidad en Amazonas, que se encuentra en la calle Carvalho Leal, 555, distrito -. Cachoeirinha, en la ciudad de Manaus/AM, Brasil. La muestra para el estudio fue através de 15 proyectos internos, donde 8 proyectos son de innovación industrial, 4 proyectos son de innovación medioambiental y 3 proyectos de innovación automovilística. El procedimiento metodológico utilizado consiste en tres fases: la primera se realizó mediante la revisión de fuentes bibliográficas donde fue posible conceptualizar la innovación y establecer sus ámbitos de aplicación; vincular la innovación para el entorno industrial; describir los principales modelos existentes, la innovación abierta y la innovación cerrada. 
La segunda fase tiene como objetivo contextualizar el estudio de caso de 15 proyectos internos de innovación analizados; determinar los 15 proyectos internos y entregar los datos de la encuesta de los equipos, los autores y año de finalización de cada proyecto; presentar y discutir los resultados. Para Gummesson (2014), este tipo de encuesta puede dar más validez y relevancia a la investigación, ya que se concentra en los resultados, los detalles del proceso de investigación, técnicas para aumentar la fiabilidad y técnicas para aumentar la precisión de las informaciones colectadas.

La tercera fase busca identificar los posibles escenarios interiores en el que se produjeron la creación y producción de los proyectos internos para presentar una nueva metodología con quince etapas para trabajar la innovación o mejoría de productos. Una vez que los proyectos no siguen un modelo formal de la innovación, fue necesario analizar la situación de cada grupo de los 15 proyectos a partir del modelo de innovación abierta, con una división del proceso de innovación en tres fases básicas: concepto, desarrollo y mercado.

En la fase de concepto se comprobó si los proyectos de innovación industrial, medioambiental y automovilística tienen alianzas estratégicas que se extiende más allá de sus fronteras con asociaciones de desarrollo. En la etapa de desarrollo se comprobó si los proyectos utilizan criterios para mitigar las fallas y detectar errores. Si los proyectos permiten que los conocimientos o tecnologías puedan fluir libremente en cualquier etapa. En el escenario de mercado se verificó si los proyectos se centran en la propuesta de valor al cliente y mezcla ideas internas y externas como una oportunidad para aumentar la innovación.

\section{LA INNOVACIÓN INDUSTRIAL, CONCEPTO Y SU ALCANCES}

El Manual de Oslo (2005) define la innovación como un producto nuevo o mejorado, un método de comercialización y también como un método organizativo que ha sido absorbida por la organización y que está en funcionamiento. Conocer el concepto de innovación industrial y su amplitud es importante para entender el funcionamiento, aplicación y metodología.

El pionero en el estudio de la innovación fue el austríaco Joseph Alois Schumpeter, quien a mediados de los años 50 señaló que la productividad y el crecimiento de una organización dependen de cambios técnicos. Esto significa que las empresas que invierten en innovación tecnológica, generarían los factores decisivos para el crecimiento (Teulon, 2014). Estos nuevos ajustes han sido estratégicas para la aparición de nuevos conceptos de innovación y su alcance. En el ámbito administrativo, Hwang (2004), declara que la innovación necesita tener un enfoque directo en proyectos a partir de una estructura adecuada para la organización. Para Birkinshaw et al. (2008), la innovación es la implementación de una práctica de gestión que abarca todo el proceso de organización y destina alcanzar nuevos objetivos de la organización. Para Mol et al. (2009), la innovación debe ser una práctica de gestión de proyectos cuyo objetivo es mejorar el desempeño de la empresa en conjunto. Para Battisti y Stoneman (2010), la innovación implica nuevas prácticas, nuevos conceptos de mercadeo y nuevos proyectos con estrategias empresariales. Para Damanpour y Aravind (2012), la innovación es un nuevo enfoque de la administración, lo que produce un cambio en la estrategia, los procedimientos administrativos y el sistema de organización. Siguiendo este mismo concepto, Rowley et al. (2011), aporta otro enfoque, más allá de los temas ya presentados anteriormente, como productos innovadores y servicios, la producción o proceso, es decir, los autores introducen la invocación de las personas. Esta innovación comienza con el foco en el individuo, aprovechando su experiencia creativa para aumentar el nivel de los proyectos internos de las organizaciones.

La persona sola o en equipo, con pleno conocimiento del proceso de producción, desarrolla habilidades creativas con la ayuda de las herramientas adecuadas y de una metodología sistémica, contribuyendo a la mejora continua y la generación de nuevas ideas. Para Manso (2011), el trabajo en equipo de innovación es tan importante que las industrias deben crear incentivos para ello. La innovación es a menudo desarrollada por equipos que trabajan juntos en una investigación aplicada y, por esta razón, es interesante crear nuevas oportunidades para que este trabajo creativo pueda prosperar.

Por esta razón, la búsqueda de profesionales creativos, eficientes e innovadores ya no es una opción para convertirse en una necesidad en las industrias. El objetivo es que las personas que trabajan en equipo puedan mirar fuera de la organización, reconocer que el conocimiento está ampliamente disponible para todos y dar lugar a invenciones de consumo del mercado, lo que contribuye a la interacción entre ellos. Esta búsqueda de profesionales creativos hacen los departamentos más integrados. Según Hongxing y Pengfei (2012), el equilibrio funcional permite obtener excelentes ventajas y productos cada vez más innovadores para la sociedad. En este sentido, la creación de la colaboración entre los departamentos de la organización y el mercado externo, son incentivos necesarios para que se tengan conocimiento de la necesidad de los posibles clientes para el proceso de innovación, basado en el conocimiento para innovar. 
Estos incentivos dependen del contexto de cada empresa. El ideal no es el tamaño de la inversión, pero que estos incentivos se llevan a cabo de forma continua como parte de la cultura organizacional y que pueda motivar a los demás a obtener cualidades que conduzcan al pensamiento creativo e innovador. En este sentido, Rosenbusch (2011) afirma que, aunque la innovación puede implicar una inversión inicial, riesgos e incertidumbres, los beneficios tales como la diferenciación competitiva, la lealtad del cliente y los precios competitivos de productos innovadores exceden los costos iniciales. Según Mehta et al. (2014), mencionan que el pensamiento no tiene límites a la innovación y las empresas que planean explotar el potencial de sus empleados tienen una gran ventaja, a pesar de las presiones internas o externas. Para traer todos estos beneficios, es necesario planear estrategias para enfoques y formas de proyecto más abierto, teniendo en cuenta los mecanismos a implementar, los recursos y capacidades para ser desplegados para apoyar la innovación abierta.

\section{DESCRIPCIÓN DE LOS PRINCIPALES MODELOS DE INNOVACIÓN INDUSTRIAL}

De acuerdo con el Manual de Oslo (2005), la innovación será un éxito desde el momento en que causa cierto impacto, generando retornos financieros y riqueza. Para Bourletidis (2014), la innovación industrial debe ocurrir dentro de un modelo destinado a aumentar el valor de su producto en el mercado, generando beneficios. Modelos de innovación son, por lo tanto, de una importancia crucial para salir de las situaciones de crisis, lo que requiere un esfuerzo concertado de las tecnologías de las estructuras organizativas, las herramientas y políticas. Según Şimşit et al. (2014), para trabajar en situaciones de crisis la organización debe tener su estrategia y la planificación en detalle, con un modelo de flujo de trabajo centrado en la competitividad, siendo necesario hacer un análisis de las diversas metodologías y medidas existentes y establecer un modelo de trabajo de esta metodología. Según Kadar et al. (2014), el modelo de innovación correcta ayuda en el proceso de recuperación mediante la promoción del crecimiento y el desarrollo sostenible. La figura 01 muestra dos de estos modelos, llamados innovación cerrada e innovación abierta, propuesto por Clark y Wheelwright (1993).

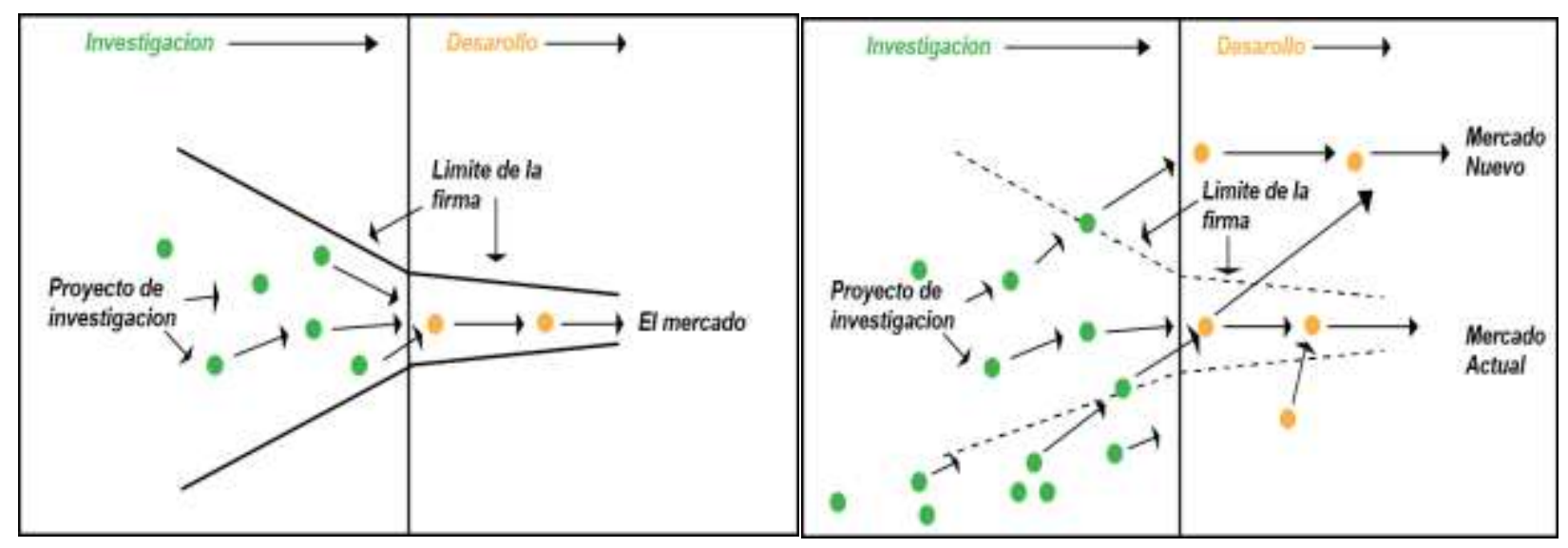

Fig. 1: Innovación cerrada y innovación abierta (adaptado de Clark y Wheelwright 1993)

Este sistema puede ser ejemplificado por un embudo de etapas en el proceso de innovación. El embudo tiene como entradas las ideas que se conviertan en proyectos, entonces los proyectos en negocios. El éxito se define estrictamente como un nuevo producto, la tecnología o mercado para la compañía. La innovación del embudo es un modelo lineal que describe un conjunto de varios pasos requeridos para alcanzar el desarrollo del producto. Todas las ideas se filtran, para conseguir lo mejor para el mercado receptor. La innovación abierta, propuesta por Chesbrough (2006,) es un modelo de innovación que funciona similar al modelo lineal, sin embargo, está cambiando con su entorno exterior, la creación de nuevos conocimientos y mejores oportunidades para los mercados de consumo. Uno de estos nuevos puntos de vista puede haber crecimiento y desarrollo de las telecomunicaciones, que crean un ambiente favorable para la expansión de este tipo de innovación abierta. Para Nylén y Holmström (2015), el uso de la tecnología digital ofrece a las industrias la oportunidad estratégica para negociar con los clientes para compartir sus intenciones y preferencias a fin de obtener conocimientos sobre los patrones de uso y de compra.

Cada tipo de innovación debería generar algún valor, por lo tanto, la base de todo comienza en conocimiento del mercado y de los clientes. Según Dahlander y David (2010), la innovación abierta debería establecer un análisis desde la perspectiva del ciclo de vida del producto y las diferentes fases que evoluciona desde el concepto hasta la comercialización. Este efecto se debe a que el mercado externo está cambiando constantemente, es decir, cuanto mayor es la incertidumbre, más conservador serán los proyectos de innovación para este mercado y mayores serán las posibilidades de trabajar con la innovación abierta. La innovación abierta lleva a la organización a evolucionar desde el paradigma de la competencia con el 
paradigma de la colaboración de todos en la búsqueda de la excelencia. Sin lugar a dudas, la innovación abierta tiene como objetivo resultados impresionantes. Para Huizingh (2011), la innovación abierta es un concepto que encaja muy bien con muchas tendencias en la gestión. La innovación abierta se presenta de muchas formas lo que aumenta el concepto de riqueza. Por lo tanto, el desarrollo de estructuras de estos tipos de innovaciones es necesario.

\section{RESULTADOS Y DISCUSIÓN}

La Tabla 1 muestra los 15 proyectos internos de innovación del SENAI desarrollados por los equipos en el año 2014. Todos los proyectos están dirigidos a la mejora de productos. Para la protección de los secretos comerciales, se puso a disposición sólo resumen superficial de cada proyecto de innovación, sin entrar en más detalles técnicos.

Tabla 1: Los quince proyectos de innovación

\begin{tabular}{|c|c|c|c|}
\hline Tema & Tipos de proyectos & Autores & Resumen \\
\hline $\begin{array}{l}\text { Gato para } \\
\text { elevación }\end{array}$ & $\begin{array}{l}\text { La mejora del producto } \\
\text { área automovilística }\end{array}$ & $\begin{array}{l}\text { Jozielber Valente, Jonas Silva, } \\
\text { Jefeson Leite, Jonas Veiga }\end{array}$ & $\begin{array}{l}\text { Prototipo de máquina para máquinas } \\
\text { de reparación de neumáticos }\end{array}$ \\
\hline Monta silla fácil & $\begin{array}{l}\text { La mejora del producto } \\
\text { área de medio ambiente }\end{array}$ & $\begin{array}{l}\text { Gabriel Martins, Hyan Brendell, } \\
\text { Jorleylson Malheiros }\end{array}$ & $\begin{array}{l}\text { Silla prototipo con un montaje rápido } \\
\text { a través de ranuras }\end{array}$ \\
\hline $\begin{array}{l}\text { Tufadeira } \\
\text { neumática }\end{array}$ & $\begin{array}{l}\text { La mejora del producto } \\
\text { área industrial }\end{array}$ & $\begin{array}{l}\text { Caíque da Silva, Fábio Cruz, } \\
\text { José Luiz, Willison Ferreira }\end{array}$ & $\begin{array}{l}\text { Prototipo para escobas a través de la } \\
\text { configuración automática }\end{array}$ \\
\hline $\begin{array}{l}\text { Latas } \\
\text { compactador }\end{array}$ & $\begin{array}{l}\text { La mejora del producto } \\
\text { área de medio ambiente }\end{array}$ & $\begin{array}{l}\text { Lucilo Joel, Romal Ramon, } \\
\text { Sirney Santos, Stefano Castro }\end{array}$ & $\begin{array}{l}\text { Prototipo deformación automatizado } \\
\text { de latas de aluminio }\end{array}$ \\
\hline $\begin{array}{l}\text { Máquina de pelar } \\
\text { la naranja }\end{array}$ & $\begin{array}{l}\text { La mejora del producto } \\
\text { área industrial }\end{array}$ & $\begin{array}{l}\text { Bruno Costa, Patrick Coelho, } \\
\text { Wellington Machado }\end{array}$ & $\begin{array}{l}\text { Máquina prototipo con extracción } \\
\text { automática }\end{array}$ \\
\hline $\begin{array}{l}\text { Soporte para } \\
\text { motores diesel }\end{array}$ & $\begin{array}{l}\text { La mejora del producto } \\
\text { área automovilística }\end{array}$ & $\begin{array}{l}\text { Marcos Ariel, Martiniano Alves, } \\
\text { Raphael de Oliveira }\end{array}$ & $\begin{array}{l}\text { Sistema automático para } \\
\text { mantenimiento de motores diesel }\end{array}$ \\
\hline $\begin{array}{l}\text { Soporte de } \\
\text { motocicletas }\end{array}$ & $\begin{array}{l}\text { La mejora del producto } \\
\text { área automovilística }\end{array}$ & $\begin{array}{l}\text { André Silva, Greice Santos, } \\
\text { Johnes Oliveira, WarlenPontes }\end{array}$ & $\begin{array}{l}\text { Sistema de elevación automática } \\
\text { para los servicios de mantenimiento }\end{array}$ \\
\hline $\begin{array}{l}\text { Extractor de } \\
\text { disyuntores }\end{array}$ & $\begin{array}{l}\text { La mejora del producto } \\
\text { área industrial }\end{array}$ & $\begin{array}{l}\text { Lucio Miranda, Gilfran } \\
\text { Carvalho, Izaque Duarte }\end{array}$ & $\begin{array}{l}\text { Sistema para desmantelamiento de } \\
\text { los disyuntores industriales. }\end{array}$ \\
\hline $\begin{array}{l}\text { Máquina para } \\
\text { Ilevar açai }\end{array}$ & $\begin{array}{l}\text { La mejora del producto } \\
\text { área de medio ambiente }\end{array}$ & $\begin{array}{l}\text { Armando Guedes, Elielton } \\
\text { Silva, Ericson Soares }\end{array}$ & $\begin{array}{l}\text { Prototipo máquina automática para } \\
\text { el procesamiento de açai. }\end{array}$ \\
\hline $\begin{array}{l}\text { Prótesis } \\
\text { ortopédica }\end{array}$ & $\begin{array}{l}\text { La mejora del producto } \\
\text { área industrial }\end{array}$ & $\begin{array}{l}\text { Bianca Marinho, Lucenilva } \\
\text { Castro }\end{array}$ & $\begin{array}{l}\text { Prototipo de una prótesis mecánica } \\
\text { para las personas con incapacidad }\end{array}$ \\
\hline $\begin{array}{l}\text { Seguro } \\
\text { mecánica }\end{array}$ & $\begin{array}{l}\text { La mejora del producto } \\
\text { Área industrial }\end{array}$ & $\begin{array}{l}\text { Cleber Roger, Jacqueline } \\
\text { Santos, Rodolfo Rocha }\end{array}$ & $\begin{array}{l}\text { Sistema mecánico de bloqueo y } \\
\text { desbloqueo }\end{array}$ \\
\hline $\begin{array}{l}\text { andamios } \\
\text { automáticos }\end{array}$ & $\begin{array}{l}\text { La mejora del producto } \\
\text { área industrial }\end{array}$ & $\begin{array}{l}\text { Arlison George, Francisco } \\
\text { Leonardo, Françoizy Alves }\end{array}$ & $\begin{array}{l}\text { Prototipo con sistema de elevación } \\
\text { automática }\end{array}$ \\
\hline $\begin{array}{l}\text { barbacoa } \\
\text { automatizada }\end{array}$ & $\begin{array}{l}\text { La mejora del producto } \\
\text { área de medio ambiente }\end{array}$ & $\begin{array}{l}\text { Jonatas da Silva, Wagner de } \\
\text { Oliveira }\end{array}$ & $\begin{array}{l}\text { Sistema de movimiento automático y } \\
\text { ventilación mecánica }\end{array}$ \\
\hline $\begin{array}{l}\text { Horno para } \\
\text { tostar harina }\end{array}$ & $\begin{array}{l}\text { La mejora del producto } \\
\text { área industrial }\end{array}$ & $\begin{array}{l}\text { Ana Carolina, Thais Leal, Erika } \\
\text { Carvalho, Vanderlan Carvalho }\end{array}$ & $\begin{array}{l}\text { Rotación automática de la harina } \\
\text { tostada }\end{array}$ \\
\hline $\begin{array}{l}\text { Alimentador } \\
\text { automático para } \\
\text { perros. }\end{array}$ & $\begin{array}{l}\text { La mejora del producto } \\
\text { área industrial }\end{array}$ & $\begin{array}{l}\text { Maria da Conceição, Luiz } \\
\text { Henrique, Diego Moreira, Luiz } \\
\text { Carlos, Rildo Lima }\end{array}$ & Prototipo con sistema de sensores \\
\hline
\end{tabular}


Se puede observar que ocho (08) proyectos pertenecen a la mejora industrial y contempla un producto existente, que combina los beneficios de la funcionalidad, la seguridad, la operatividad y la comodidad. El factor planteado se refiere a indicar que estos productos mejorados forman parte de los miembros de estos equipos de medio ambiente de trabajo. Este factor es importante porque según Taveira, et al. (2014), la experiencia y los conocimientos adquiridos por los miembros de estos equipos pueden ser valorado en los proyectos de innovación, convirtiéndose en otro punto estratégico para las organizaciones.

El área del medio ambiente representó de cuatro (4) productos que se han mejorados significativamente. La reutilización de materiales de desecho antes en la naturaleza era el hilo común entre todos los proyectos desarrollados por los alumnos. Para Allacker et al. (2014), estas iniciativas son positivas porque varios materiales hoy en día tienen la característica que pasar por el proceso de recuperación y volver a utilizar de nuevo para el consumo y la producción sostenibles. Para Setyowati (2014), la conciencia mundial sobre el medio ambiente comenzó a aumentar en los últimos años. Estas innovaciones ambientales garantizan el desarrollo de nuevos productos que pueden ser desmontados fácilmente, contribuyendo así a una mayor rentabilidad. La industria automovilística representó el tres (3) proyectos en el área de mantenimiento y servicios. Para Ryley et al. (2014), es importante que la industria automovilística evolucione continuamente en tecnología y mejoras porque los estudios técnicos y el desarrollo de tecnologías son alternativas que involucra directamente a la sostenibilidad del medio ambiente.

Se hizo hincapié en el desarrollo del concepto de un producto para el mercado exterior, a pesar de los equipos que tienen un clima favorable para el uso interno de sus propias empresas orientadas a la innovación. De acuerdo con la figura 2, de 08 (ocho) de los proyectos de áreas industriales que contemplan la mejora de un producto existente, sólo tres (03) se trasladaron a la etapa de la viabilidad del desarrollo y la producción. Las principales barreras fueron: ausencia de una extensa investigación previa, los proyectos con bajo valor agregado, la falta de experiencia en el área de proyectos de equipo. Estos son puntos administrativos importantes porque según Espinosa, Dias y Back (2008), hacen parte del planeamiento para que la organización tenga una alta probabilidad de éxito en la implementación de la innovación.

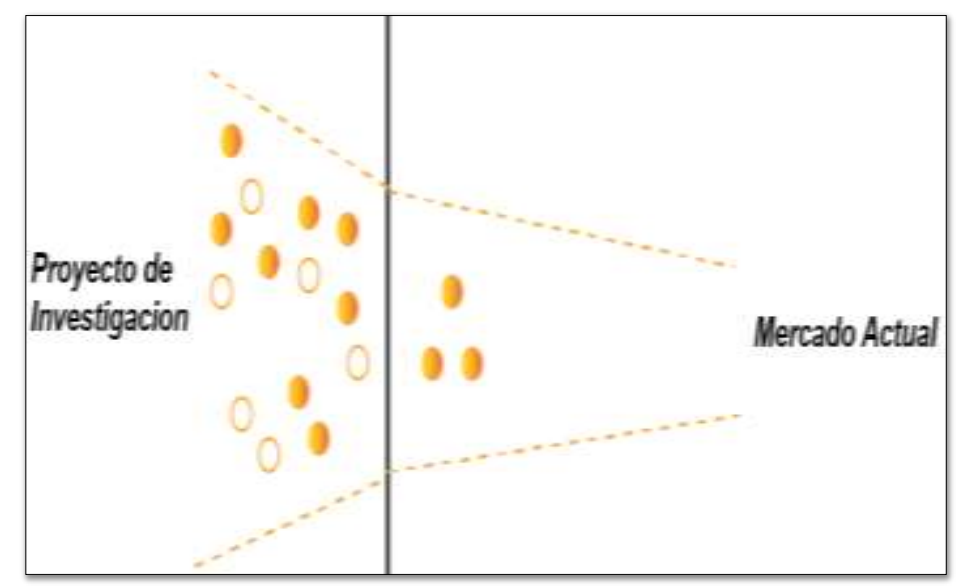

Fig. 2: Proyectos aprobados a partir del modelo de innovación abierta (adaptado de Chesbrough, 2006)

De 03 (tres) proyectos restantes, ninguno fue a la fase de fabricación y comercialización posterior. Las barreras presentadas fueron: la falta de diseño mecánico, la falta de diseño eléctrico de los sistemas, la falta de sistema de producción y distribución de los productos y componentes, la falta de garantía y piezas de la vida y la sustitución, la eficiencia y pruebas de eficacia. Para Iván y Despa (2014), la fase de planificación debe garantizar la adecuada metodología, cronograma, presupuesto y personal para un proyecto innovador. Todos los factores antes mencionados tendrán un papel crucial en la implementación de un proyecto innovador.

Los proyectos de áreas ambientales fueron diseñados para contribuir al desarrollo de nuevos productos con mejoras significativas en la sociedad, con énfasis en los nuevos materiales de materiales, reutilización y eco. De acuerdo con la figura 3, de cuatro (04) proyectos del área de medio ambiente, sólo dos (02) pasa a la etapa de factibilidad de producción. Las barreras fueron: falta de clientes interesados en los productos, la relación con la sociedad y otras instituciones ambientales, el conocimiento de la legislación ambiental, la falta de investigación previa, se proyecta con bajo valor agregado. 


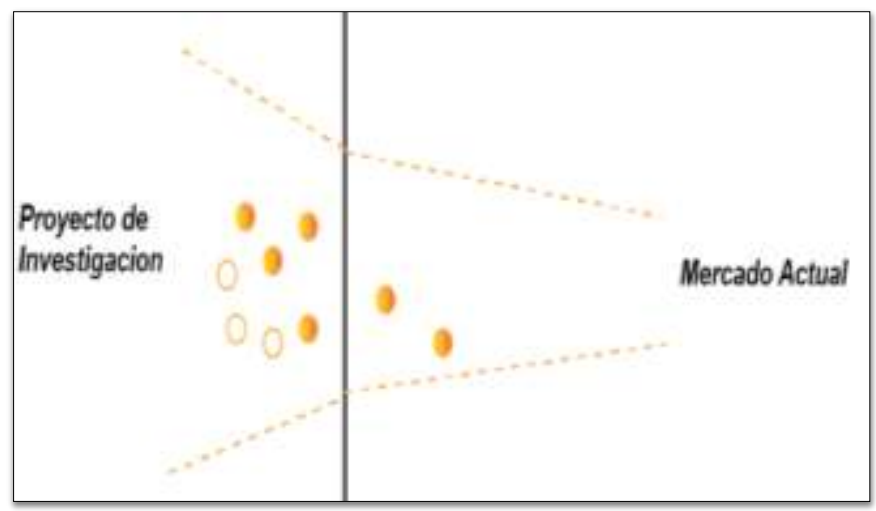

Fig. 3: Proyectos aprobados a partir del modelo de innovación abierta (adaptado de Chesbrough, 2006)

Del resto, ningún proyecto ha pasado a la fase de fabricación y comercialización posterior. Las barreras fueron: los altos costos de los proyectos, desarrollo de diseño mecánico y eléctrico, sistema de producción y distribución, garantía y piezas de la vida y la sustitución, la eficiencia y la eficacia de las pruebas. Para Prendeville, et al. (2014), las partes interesadas externas son tan importantes como el proyecto, ya que juegan un papel crucial, proporcionando información y recomendaciones importantes que servirá de ayuda para el desarrollo de nuevos productos ambientales y los nuevos materiales. Según Peças et al. (2014), a través de la gestión de los costos del proyecto y el ciclo de vida de los productos, los impactos ambientales y el rendimiento en el trabajo técnico con diferentes alternativas, lo que permite al equipo a elegir la mejor alternativa de acuerdo con su práctica y estrategia corporativa. Para Cheng et al. (2014), el rendimiento del negocio está directamente e indirectamente afectada por el entorno de la organización, y una organización con las preocupaciones ambientales también influye en el desarrollo de nuevos productos ambientales.

De acuerdo con la figura 4, de tres (03) proyectos presentados en el segmento automotriz, sólo uno (01) pasó a la etapa de factibilidad de producción. Las barreras encontradas fueron la falta de experiencia de algunos miembros del equipo, la falta de investigación previa, la falta de proyectos de clientes de bajo valor añadido.

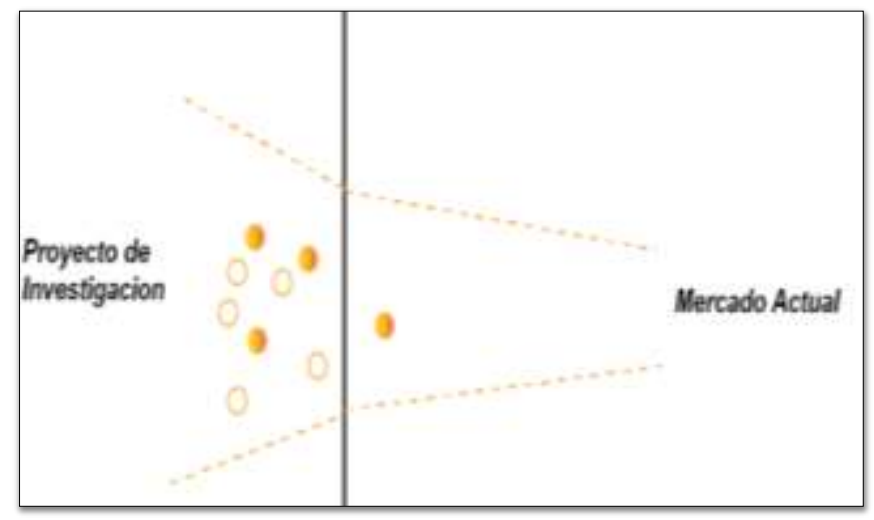

Fig. 4: Proyecto aprobado a partir del modelo de innovación abierta (adaptado de Chesbrough, 2006)

Del resto, ningún proyecto ha pasado a la fase de fabricación y comercialización posterior. Las barreras fueron: diseño de producto, diseño mecánico, sistema de producción y distribución eléctrica, garantía y piezas de la vida y la sustitución, la eficiencia y pruebas de eficacia. Para Sinkovics et al. (2014), los proyectos de innovación necesitan para conectar las necesidades de desarrollo económico de las comunidades locales para tener un impacto positivo y en consecuencia ganar futuros consumidores por el producto.

\section{RESULTADOS}

Con los resultados de las reuniones llevadas a cabo con los técnicos, se obtuvo los principales obstáculos encontrados en el desarrollo de los proyectos. Son ellos: 1) Clientes: no fueron exigidos, es decir, no tenía ninguna asociación para apoyar el desarrollo del proyecto y en consecuencia, el intercambio de experiencias e información entre las personas. 2) Alianzas: no se llevó a cabo ninguna perspectiva con las instituciones, organizaciones no gubernamentales, empresas públicas y privadas, universidades e institutos de tecnología a las asociaciones estratégicas. 3) La gente: los miembros del equipo no tenían cualificaciones o experiencia previa en el proceso de desarrollo de productos. No tenían una oficina o lugar con su propio equipo, consolidada para resolver las dudas relacionadas con los proyectos. 4) Medio Ambiente: el medio ambiente tenía pocos incentivos para la innovación práctica y la participación en concursos y retos. Hay laboratorios y 
talleres con máquinas y equipos avanzados, sin embargo, el enfoque sigue siendo en los servicios y no en la innovación. 5) Mercado: no se realizó con anterioridad la investigación y tendencias de proyección y de consumo basados en encuestas estadísticas para el consumo de los productos y la lealtad del cliente. 6) Diseñador: Los profesionales que trabajaron sólo la primera vez con los equipos. 7) Gestión: No se presentó documentos o procedimientos como un guía inicial de las acciones de los equipos de los proyectos internos.

Teniendo identificados las dificultades existentes, fue preparado los quince pasos para establecer las directrices, organizar eficazmente los proyectos internos de innovación, promover y sistematizar las actividades, promover el flujo de información para generar conocimiento, aumentar la competitividad y la excelencia de la organización y promover la cultura de la innovación en la empresa SENAI. Además, de acuerdo con la figura 5, se elaboró un diagrama de flujo del proceso de innovación con los quince pasos. Este modelo llamado de Modelo Vida, ahora ha contribuido con las dificultades de gestión que se encuentran principalmente los proyectos internos en el principio, con la elección de los aspectos que deben ser controlados y sus criterios de visibilidad que le ayudarán en la toma de decisiones en cada etapa del proceso.

\begin{tabular}{|c|c|c|}
\hline \multicolumn{3}{|c|}{ 15. Clientes o Mercado } \\
\hline \multicolumn{3}{|c|}{ 14.Producción y la Calidad del Piloto } \\
\hline \multicolumn{3}{|c|}{ 13.Mercadeo de Producto } \\
\hline & 12. Riesgos & \\
\hline & 11.Propiedad Intelectual & \\
\hline & 10.Prototipo & \\
\hline & 9.Costos & \\
\hline & 8.Suministros & \\
\hline & 7.Viabilidad Técnica & \\
\hline & 6.Proyectos & \\
\hline 3.Personas & 4.Procedimientos & 5.Ambiente \\
\hline \multicolumn{3}{|c|}{ 2.Gestión y la Alta Dirección } \\
\hline \multicolumn{3}{|c|}{ 1.Clientes o Mercado } \\
\hline
\end{tabular}

Fig. 5: VIDA, nuevo modelo para la innovación de proyectos internos

En consideración a los problemas encontrados, se rediseñó el proceso sobre la base de 1) Los clientes o mercados: los proyectos internos del SENAI deben establecer los clientes potenciales con prioridad a las demandas, así como la exploración de la innovación en nuevos mercados, sea un mercado nacional o internacional. Para Ahern y Harford (2014), las empresas solo funcionan se estuvieren conectadas con los clientes. 2) Gestión y alta dirección: la empresa debe establecer la responsabilidad y compromiso con el desarrollo y el progreso de la gestión de todo el proceso de innovación y comunicación eficaz a toda la organización. Para Agostino et al. (2015) los cambios en la organización y los efectos de la globalización mundial exigen que la alta dirección de las empresas mejore su gestión de procesos y proyectos. 3) Personas: los profesionales y empleados deben tener niveles adecuados de formación, habilidades y experiencia para actividades de innovación profesional y académico. Para Liu et al. (2014), la intensidad de habilidad interna es crucial para las actividades de innovación de las empresas. 4) Procedimientos: la empresa debe establecer procedimientos y documentos de funcionamiento de todos los procesos necesarios para la gestión de los proyectos de innovación, la identificación de los procesos y la secuencia de la interacción con ellos, con métodos y criterios adecuados para su perfecto funcionamiento. De acuerdo con De Oca et al. (2015), los procedimientos son esenciales para que una empresa pueda alcanzar sus objetivos de negocio. La calidad de los procedimientos adoptados tiene un impacto significativo en el desarrollo de cualquier empresa. 5) Medio Ambiente: la empresa debe disponer de instalaciones adecuadas, ofreciendo factores físicos y humanos para el desarrollo de actividades de innovación. Debe tener en cuenta la infraestructura de maquinaria, equipo, programa de informática y materiales de apoyo a la práctica y el desarrollo de actividades de innovación. Para Fagerholm et al. (2015), el rendimiento del equipo que trabaja con proyectos de innovación es cada vez más relacionada con el medio ambiente que vive en constante cambio. Excelente rendimiento es una construcción dinámica que depende del ambiente de trabajo en el que opera el equipo. 6) Proyectos: etapa de entrada, 
donde se muestran los nuevos proyectos en detalles. 7) Viabilidad técnica: los proyectos deben establecer la construcción de estudio técnico del producto con el uso descriptivo de las tecnologías existentes. 8) Suministros: los proyectos deben demostrar el poder de negociación con los proveedores y cómo van a procesar los elementos para ser manejados, movidos, almacenados, procesados y transportados por la logística. 9) Costos: los proyectos deben desarrollar un estudio que demuestra la viabilidad económica de todos los escenarios que la innovación tiene que ser generado. 10) Prototipo: deben desarrollar el prototipo que puede ser visual o material, representando la originalidad del nuevo producto para medir su eficacia. 11) Propiedad intelectual: la empresa debe ayudar los con un estudio detallado internacionalmente sobre la búsqueda de la novedad y de la protección de los derechos relacionados con el proyecto de innovación y su patente. 12) Riesgos: la empresa debe hacer un estudio compacto que describe los riesgos inherentes al proyecto, el impacto y plan de acción. 13) Mercadeo de producto: la empresa debe establecer un estudio detallado de diseño del producto y el mercado. 14) La producción piloto y calidad: deben hacer las pruebas de fabricación y control de calidad del producto. 15) Clientes o mercados: deben tener como prioridad el regreso de la satisfacción del cliente y la información generada. Todos estos factores combinados con la falta de madurez y experiencia es en gran parte, según Espinosa y Salinas (2010), los principales problemas que redundan de forma adversa al momento de querer introducir innovaciones en el proceso.

\section{CONCLUSIONES}

Se concluye que la optimización del proceso de innovación con los proyectos internos abarcando los quince pasos, ha logrado elevar significativamente el nivel de aceptación por las empresas del Polo Industrial de Manaus (PIM), reposicionando el SENAI como promotor de la innovación y transferencia de tecnología.

Además se pueden obtener las siguientes conclusiones:1) los resultados alcanzados a la fecha estimula la empresa a avanzar no desarrollo del proceso de innovación eficiente de los proyectos internos con la integración de la teoría y práctica, estimulando otras empresas, industrias y organizaciones; 2) el nuevo modelo presenta una contribución para los proyectos de innovación ya que sugiere un método real de abordaje; 3) el modelo es simples, fácil de utilizar y contempla todos los principales puntos para desarrollar un proyecto interno.

Es importante decir que el SENAI ya cuenta con la certificación ISO 9001:2008, donde logró muchos beneficios con la completa integración de sus procesos internos con los quince pasos. Así, otras organizaciones que tienen la certificación medioambiental: ISO 14000, genérica de las normas ambientales e ISO 14001 específica de la gestión ambiental en las empresas también son beneficiadas en la mejora corporativa de sus productos y clientes.

Sólo una gran idea no es garantía de éxito de un nuevo producto. El proceso de innovación es parte de un todo. El modelo VIDA presenta puntos importantes que pueden servir como incentivo para todas las empresas que deseen iniciar o desea sistematizar la innovación y la creatividad de sus proyectos en su proceso interno.

\section{REFERENCIAS}

Agostino, M., Giunta, A., Nugent, J. B., Scalera, D., y Trivieri, F. The importance of Being a Capable Supplier: Italian Industrial Firms in Global Value Chains, doi: 10.1177/0266242613518358, International Small Business Journal, 33: 708-730 (2015)

Ahern, K. R., y Harford, J. The Importance of Industry Links in Merger Waves. The Journal of Finance, 69(2), 527-576. (2014)

Allacker, K., Mathieux, F., Manfredi, S., Pelletier, N., De Camillis, C., Ardente, F., y Pant, R. Allocation Solutions for Secondary Material Production and end of Life Recovery: Proposals for Product Policy Initiatives, doi:10.1016/j.resconrec.2014.03.016, Resources, Conservation and Recycling, 88, 1-12 (2014)

Battisti, G., y Stoneman, P. How innovative are UK firms? Evidence From the Fourth UK Community Innovation Survey on Synergies Between Technological and Organizational Innovations, doi: 10.1111/j.14678551.2009.00629.x, British Journal of Management, 21(1), 187-206, (2010)

Birkinshaw, J., Hamel, G., y Mol, M. J. Management Innovation, doi:10.5465/AMR.2008.34421969, Academy of Management Review, 33(4), 825-845, (2008)

Bourletidis, D. The Strategic Model of Innovation Clusters: Implementation of Blue Ocean Strategy in a Typical Greek Region, doi:10.1016/j.sbspro.2014.07.093, Procedia-Social and Behavioral Sciences, 148, 645-652, (2014) 
Cheng, C. C. Yang, C. L., y Sheu, C. The Link Between Eco-Innovation and Business Performance: a Taiwanese Industry Context, doi:10.1016/j.jclepro.2013.09.050, Journal of Cleaner Production, 64, 81-90, (2014)

Chesbrough, Henry William. Open Innovation: The New Imperative for Creating and Profiting From Technology. Harvard Business Press, (2006)

Clark, K. B. y Wheelwright, S. C. Managing New Product Development - Text and Cases. $1^{\circ}$ edición, books.google, 291 - 336, Harvard Business School, (1993)

Dahlander, L. y Gann, D. M. How Open is Innovation? doi:10.1016/j.respol.2010.01.013, Research Policy, 39(6), 699-709, (2010)

Damanpour, F. y Aravind, D. Managerial Innovation: Conceptions, Processes, and Antecedents, doi: 10.1111/j.1740-8784.2011.00233.x, Management and Organization Review, 8(2), 423-454, (2012)

De Oca, I. M. M., Snoeck, M., Reijers, H. A., y Rodriguez-Morffi, A. A Systematic Literature Review of Studies on Business Process Modeling Quality, doi:10.1016/j.infsof.2014.07.011, Information and Software Technology, 58, 187-205, (2015)

Espinosa, F. F., Dias, A., y Back, N. Un Procedimiento de Evaluación de las Condiciones Necesarias para Innovar la Gestión de Mantenimiento, doi: 10.1612/inf.tecnol.4313it.09, Información Tecnológica, Vol. 19(1), 97-104 (2008)

Espinosa, F. F., y Salinas, G. E. Evaluación de la Madurez de la Función Mantenimiento para Implementar Innovaciones en su Gestión, doi: 10.1612/inf.tecnol.4313it.09, Información tecnológica, 21(3), 3-12. (2010)

Fagerholm, F., Ikonen, M., Kettunen, P., Münch, J., Roto, V., y Abrahamsson, P. Performance Alignment Work: How Software Developers Experience the Continuous Adaptation of Team Performance in Lean and Agile Environments, doi:10.1016/j.infsof.2015.01.010, Information and Software Technology, 64, 132-147, (2015)

Genis-Gruber, A., \& Öğüt, H. Environmental Factors Affecting Innovation Strategies of Companies: Customers and Suppliers Effect, doi:10.1016/j.sbspro.2014.09.033, Procedia-Social and Behavioral Sciences, 150, 718725, (2014)

Gündoğdu, M. Ç. Re-thinking Entrepreneurship, Intrapreneurship, and Innovation: Multi-concept Perspective, doi:10.1016/j.sbspro.2012.04.034, Procedia-Social and Behavioral Sciences, 41, 296-303, (2012)

Gummesson, E. Service Research Methodology: From Case Study Research to Case Theory, doi: 10.5585/riae.v13i4.2180, Revista Ibero-Americana de Estratégia, 13(4), 08-17, (2014)

Huizingh, E. K. Open Innovation: State of the Art and Future Perspectives doi:10.1016/j.technovation.2010.10.002, Technovation, 31(1), 2-9, (2011)

Hwang, A. S. (2004). Integrating Technology, Marketing and Management Innovation. Research-Technology Management, 47(4), 27-31, (2004)

Hongxing, C., y Pengfei, Y. Multi-firms Product Innovation Diffusion Engineering Model in Dynamical Competition, doi:10.1016/j.sepro.2011.11.033, Systems Engineering Procedia, 3, 297-306, (2012)

Ivan, I., y Despa, M. L. (2014). Statistical Indicators for Measuring Innovation in IT. Project Management, doi: 10.1016/S2212-5671(14)00290-1, Procedia Economics and Finance, 10, 167-177, (2014)

Kadar, M., Moise, I. A., y Colomba, C. Innovation Management in the Globalized Digital Society, doi:10.1016/j.sbspro.2014.07.560, Procedia-Social and Behavioral Sciences, 143, 1083-1089, (2014)

Liu, X., Hodgkinson, I. R., y Chuang, F. M. (2014). Foreign Competition, Domestic Knowledge Base and Innovation Activities: Evidence From Chinese High-tech Industries, doi:10.1016/j.respol.2013.11.005, Research Policy, 43(2), 414-422, (2014)

Mahdi, R., Sukarman, S. S., y Yok, M. C. K. (2015). Fostering Creativity Through Innovation Engagement in Science and Technology Education: Case Study of Universiti Teknologi MARA Students, doi:10.1016/j.sbspro.2014.12.671, Procedia-Social and Behavioral Sciences, 167, 256-260, (2015) 
Manual, O. The Measurement of Scientific and Technological Activities. Proposed Guidelines For Collecting and Interpreting Innovation Data, (2005)

Manso, G. Motivating Innovation, doi: 10.1111/j.1540-6261.2011.01688.x, The Journal of Finance, 66(5), 1823-1860, (2011)

Mâţă, L., y Suciu, A. I. Exploring the Possibilities of Curriculum Innovation in Initial Language Teacher Education, doi:10.1016/j.sbspro.2014.01.748, Procedia-Social and Behavioral Sciences, 116, 3281-3285, (2014)

Mehta, M., Chandani, A., \& Neeraja, B. Creativity and Innovation: Assurance for Growth, doi: 10.1016/S22125671(14)00244-5, Procedia Economics and Finance, 11, 804-811, (2014)

Mihaela, M., \& Tiţan, E. Education and Innovation in the Context of Economies Globalization, doi: 10.1016/S2212-5671(14)00667-4, Procedia Economics and Finance, 15, 1042-1046, (2014)

Mol, M. J., y Birkinshaw, J. The Sources of Management Innovation: When Firms Introduce New Management Practices, doi:10.1016/j.jbusres.2009.01.001, Journal of Business Research, 62, 1269-1280, (2009)

Nylén, D., y Holmström, J. Digital Innovation Strategy: A Framework for Diagnosing and Improving Digital Product and Service Innovation, doi:10.1016/j.bushor.2014.09.001, Business Horizons, 58(1), 57-67, (2015)

Peças, P. Ribeiro, I., y Henriques, E. Life Cycle Engineering for Materials and Technology Selection: Two Models, One Approach, doi:10.1016/j.procir.2014.06.073, Procedia CIRP, 15, 543-548, (2014)

Prendeville, S., O'Connor, F., y Palmer, L. Material Selection For Eco-innovation: SPICE Model, doi:10.1016/j.jclepro.2014.05.023, Journal of Cleaner Production, 85, 31-40, (2014)

Rosenbusch, N., Brinckmann, J., y Bausch, A. Is Innovation Always Beneficial? A Meta-Analysis of the Relationship Between Innovation and Performance in SMEs, doi:10.1016/j.jbusvent.2009.12.002, Journal of Business Venturing, 26(4), 441-457, (2011)

Ryley, T. J., Stanley, P. A., Enoch, M. P., Zanni, A. M., y Quddus, M. A. Investigating the Contribution of Demand Responsive Transport to a Sustainable Local Public Transport System, doi:10.1016/j.retrec.2014.09.064, Research in Transportation Economics, 48, 364-372, (2014)

Rowley, J., Baregheh, A., y Sambrook, S. Towards an Innovation-Type Mapping Tool, doi: http://dx.doi.org/10.1108/00251741111094446, Management Decision, 49(1), 73-86, (2011)

Seman, N. A. A., Zakuan, N., Jusoh, A., Arif, M. S. M., y Saman, M. Z. M. The Relationship of Green Supply Chain Management and Green Innovation Concept, doi:10.1016/j.sbspro.2012.09.1211, Procedia-Social and Behavioral Sciences, 57, 453-457, (2012)

Setyowati, E. Eco-Building Material of Styrofoam Waste and Sugar Industry Fly-ash Based on NanoTechnology, doi:10.1016/j.proenv.2014.03.031, Procedia Environmental Sciences, 20, 245-253, (2014)

Sinkovics, N., Sinkovics, R. R., y Yamin, M. The Role of Social Value Creation in Business Model Formulation at the Bottom of the Pyramid-Implications for MNEs? doi:10.1016/j.ibusrev.2013.12.004, International Business Review, 23(4), 692-707, (2014)

Şimşit, Z. T., Vayvay, Ö. y Öztürk, Ö. An Outline of Innovation Management Process: Building a Framework for Managers to Implement Innovation, doi:10.1016/j.sbspro.2014.09.021, Procedia-Social and Behavioral Sciences, 150, 690-699, (2014)

Teulon, F. Actualité des Analyses de Joseph Schumpeter. Working Papers 2014-499, Department of Research, Ipag Business School, (2014)

Van der Meer, H. Open Innovation - The Dutch Treat: Challenges in Thinking in Business Models, doi: 10.1111/j.1467-8691.2007.00433.x. Creativity and Innovation Management, 16(2), 192-202, (2007)

Von Hippel, E., De Jong, J. P., y Flowers, S. Comparing Business and Household Sector Innovation in Consumer Products: Findings From a Representative Study in the United Kingdom, doi.org/10.1287/mnsc.1110.1508, Management Science, 58(9), 1669-1681, (2012) 
